\title{
Variability analysis of device-level photonics using stochastic collocation (Conference Presentation)
}

\author{
Yufei Xing, Domenico Spina, Ang Li, Tom Dhaene, Wim Bogaerts, Univ. Gent (Belgium)
}

\begin{abstract}
Abstract

Integrated photonics, and especially silicon photonics, has been rapidly expanded its catalog of building blocks and functionalities. Now, it is maturing fast towards circuit-level integration to serve more complex applications in industry. However, performance variability due to the fabrication process and operational conditions can limit the yield of large-scale circuits. It is essential to assess this impact at the design level with an efficient variability analysis: how variations in geometrical, electrical and optical parameters propagate into components performance. In particular when implementing wavelength-selective filters, many primary functional parameters are affected by fabrication-induced variability. The key functional parameters that we assess in this paper are the waveguide propagation constant (the effective index, essential to define the exact length of a delay line) and the coupling coefficients in coupling structure (necessary to set the power distribution over different delay lines).

The Monte Carlo (MC) method is the standard method for variability analysis, thanks to its accuracy and easy implementation. However, due to its slow convergence, it requires a large set of samples (simulations or measurements), making it computationally or experimentally expensive. More efficient methods to assess such variability can be used, such as generalized polynomial chaos (gPC) expansion or stochastic collocation. In this paper, we demonstrate stochastic collocation (SC) as an efficient alternative to $\mathrm{MC}$ or gPC to characterize photonic devices under the effect of uncertainty. The idea of SC is to interpolate stochastic solutions in the random space by interpolation polynomials. After sampling the deterministic problem at a pre-defined set of nodes in random space, the interpolation is constructed. SC drastically reduces computation and measurement cost. Also, like MC method, sampling-based SC is easy to implement. Its computation cost can be further reduced by choosing proper sparse grid. Meanwhile, it offers similar high accuracy and efficiency as gPC method.

We applied SC method to analyze the variability of waveguides and directional couplers. They are both building blocks in the construction of larger photonics components such as higher-order ring resonator or Mach-Zehnder filters. These wavelength filters cannot be constructed without a proper control of waveguides and directional couplers properties. However, in silicon photonics, waveguides and directional couplers are susceptible to fabrication variations: a small change in linewidth or thickness of the core can dramatically vary effective indices and coupling coefficients.

We applied SC technique to map the variability of waveguides and directional couplers. We verified its accuracy and efficiency by comparing the results with a classic MC analysis. In the simulation, this led to a speedup of $146 \times$. We also applied the technique to analyze effective index of waveguides and directional couplers measured on waferscale. Predicted variability by SC shows good agreement with measured variability. The result of this research is not limited to coupling devices. Thanks to the flexibility in the choice of the interpolation schemes and the efficiency of sparse grid sampling to choose the collocation nodes for multiple-dimension, the proposed approach is flexible and can be applied to study a broad range of photonic devices.
\end{abstract}

